\title{
Exact multielectronic electron-concentration-dependent ground states for disordered two-dimensional two-band systems in the presence of disordered hoppings and finite on-site random interactions
}

\author{
Zsolt Gulácsi \\ Department of Theoretical Physics, University of Debrecen, H-4010 Debrecen, Poroszlay ut 6/C, Hungary
}

(Received 17 April 2003; revised manuscript received 19 June 2003; published 18 February 2004)

\begin{abstract}
We report exact multielectronic ground states dependent on electron concentration for quantum-mechanical two-dimensional disordered two-band-type many-body models in the presence of disordered hoppings and disordered repulsive finite Hubbard interactions, in fixed lattice topology considered provided by Bravais lattices. The obtained ground states lose their eigenfunction character for independent electron approximation, are perturbatively not connected to the noninteracting but disordered case, and describe a localizationdelocalization transition driven by the electron concentration, being highly degenerated and paramagnetic.
\end{abstract}

DOI: 10.1103/PhysRevB.69.054204

PACS number(s): 71.10.Hf, 67.40.Db, 71.10.Pm, 71.55.Jv

\section{INTRODUCTION}

In the real life, the crystalline state is an exception rather than a rule, ${ }^{1}$ and as a consequence, disorder exists everywhere, ranging from few impurities or interstitials in a periodic host up to the completely disordered glassy and amorphous structures, alloys, and compounds. Given by this, the effects of the disorder are intensively analyzed, ${ }^{2}$ special attention being given in the last period to two-dimensional (2D) systems, where the observation of metallic behavior in 2D high-mobility samples ${ }^{3}$ contradicting the conventional noninteracting scaling theory ${ }^{4}$ has underlined the special importance of electron-electron interactions in disordered systems, at least when its value is relatively high, ${ }^{5}$ or when the competition between disorder and interaction demands the consideration of both. ${ }^{6-12}$

Deep rooted in the difficulty of describing the effects of the disorder in a nonapproximated manner, on the theoretical side the interpretations are given almost exclusively based on approximations. In the last decade however it became clear that this is not a fortunate situation, since not only the noninteracting scaling theory has been affected by new experimental results but also other approximated schemes considered previously indisputable (between them, all aspects of the Boltzmann description even for the weak-disorder limit in the treatment of the low-temperature resistivity ${ }^{1}$ ) have been forced to be reanalyzed. Based on these facts, suggestions to follow new roads have been made, underlining that the disordered materials cannot be understood by evading the real issue, and forcing the disorder into a mold of procedures standard for ordered systems. ${ }^{1}$ Furthermore, it has been stressed that the nonperturbative view on disorder could lead to significant advancement in the understanding and description of such systems. ${ }^{10}$ At the same time, several recent developments in the field require the nonapproximated solution of the wave equation for disordered and interacting systems as a key feature for a much deeper understanding of the emerging processes, and their interpretation, especially where it is expected, or experimentally is seen, that the electrons are maintaining their long-range phase coherence and retain their wave nature, as in the case of solid grains, short wires, dots, mesoscopics, proximity to critical points, presence of long-range order, or of some kind of order in general, ${ }^{2,13}$ presence of quantum interferences, ${ }^{14}$ etc.

On this background, the first steps towards exact results for disordered systems have been made. On this line especially nonperiodic models of different types were analyzed. In these models, the nonperiodicity is considered as introducing the effect of the disorder in the Schrödinger equation, and is taken into account in different ways, for example, as nonanalytic behavior in the potential, ${ }^{15}$ incommensurate potential ${ }^{16}$ quasiperiodicity, ${ }^{17}$ topological disorder connected to tessellation, ${ }^{18}$ local bond-orientational order, ${ }^{19}$ etc.; these possibilities presenting also interdependences between them. This way of describing the disorder cannot be considered as representing the level of simple toy models only, since besides the fact that real physical systems holding such properties are known, ${ }^{2,15,16,19}$ there are concrete cases where it is also known that a such type of representation (for example, through quasiperiodicity) give analogous behavior for the system as random or disordered potentials. ${ }^{15,16}$

In $D=1$ the majority of studies leading to exact results were given for Fibanocci type of lattices, ${ }^{15,20,21}$ and the interested reader will find more extended information on the 1D subject in review papers, such as Ref. 2. For $D>1$, which is of interest in this paper, the first exact results have been obtained for quasicrystalline systems, where first, theorems dealing with structure have been formulated, such as those involving inflation rules, or Conway's theorem. . $^{2,23}$ Later on, in few cases, even exact eigenfunctions have been deduced for Penrose lattice ${ }^{22}$ in $2 D^{24-27}$ This type of lattice being a prototype of quasicrystalline systems ${ }^{28}$ clearly excedes the level of curiosity of pure mathematical character since it is related to nearest-neighbor bond-orientational order which is observed, for example, in simulation of supercooled liquids and metallic glasses ${ }^{19}$ attracting clear interest. ${ }^{29}$ In the 2D Penrose lattice, in a simplified view, flat and thin rhombuses cover the plane completely, forcing the resulting pattern to be nonperiodic and introducing disorder in the system. For these systems, in 2D exact eigenstates were obtained by Kohmoto and Sutherland ${ }^{24}$ for a strictly localized state including in the Hamiltonian on-site disor- 
dered potential as well depending on the number of bonds entering in a given site; Sutherland ${ }^{25}$ obtaining a self-similar state taking into account as well on-site potential which may have eight different values depending on the nature of the site; Arai et $_{\text {al. }}{ }^{26}$ obtaining new strictly localized states in comparison with those described in (Ref. 24); and Repetowicz et al. ${ }^{27}$ obtaining eigenstates even in the presence of plaquette-diagonal hoppings. The knowledge of these results has clarified puzzles related to the influence of the disorder in several aspects, to the extent to which only exact results can provide. We note on this line clarifications of disputes originating from the interpretation of numerical results, ${ }^{24}$ evidences for the self-similarity of some ground states in disordered systems, ${ }^{25}$ evidences for singular features of ground states in certain nonperiodical systems, ${ }^{26}$ occurrence of degeneracy proportional to the system size in eigenstates, ${ }^{26}$ scaling properties of the exact ground states, ${ }^{25}$ relative stability of the confined states on boundary conditions, ${ }^{26}$ possible existence of allowed and forbidden sites in the eigenstates, ${ }^{26}$ etc.

We underline that the above-mentioned exact eigenstates are valid only for independent electrons, e.g., they were deduced from models built up on a tight-binding Hamiltonian in $\mathbf{r}$ space describing a single electron moving on an aperiodic graph. ${ }^{17}$ Because of this reason, and in the light of the facts previously presented, it would be extremely stimulating for the field to see to what extent the deduced exact properties at independent electron level remain or are not valid in exact terms for a really multielectronic and interacting system as well in the presence of disorder. In our knowledge, exact results of this type, up to this moment, are not known.

In this paper, we report, to our knowledge, for the first time exact ground states depending on the electron concentration for multielectronic and interacting 2D systems in the presence of disorder. The ground states are paramagnetic, lose their eigenstate nature in the independent electron approximation, present properties known in the Penrose lattice (for example, strong degeneracy proportional to the system size), describe a localization-delocalization transition driven by the electron concentration, and in the localized case present clear evidence for long-range phase coherence.

The results are reported for two-band-type models. The presence of two bands does not diminish the applicability of the results since, from one side, real materials are of multiband type, and the theoretical description is given usually by projecting the multiband structure in a few-band picture, ${ }^{30}$ which is stopped only for its relative simplicity at the oneband extreme level, when this is possible. From the other side, the experimental one, several materials treated traditionally in a two-band picture have been experimentally found to contain disorder and present extremely interesting properties (as non-Fermi liquid behavior, for example ${ }^{31}$ ) whose emergence is considered connected to the presence of the disorder (see for example, Refs. 32 and 33, and cited references therein). Concerning the presence of real random systems holding two type of electrons, we mention the intense activity related to rare-earth and actinide compounds which behave as random Kondo insulators ${ }^{34,35,32}$ holding two types of electrons ( $d$ and $f$ ), whose properties are described in a fixed lattice topology, but randomly distributed Hamiltonian parameters. ${ }^{33}$

The procedure we use originates from developments leading to the first exact ground states for the periodic Andersontype models obtained at a finite value of the interaction in $1 \mathrm{D},{ }^{36-38} 2 \mathrm{D},{ }^{39-41}$ and $3 \mathrm{D},{ }^{42}$ which have been made here applicable in the disordered case as well. Our model is built up on a $2 \mathrm{D}$ graph in $\mathbf{r}$ space, whose all vertices are of the same rank (four edges are collected by every vertex), so the topology is fixed. Four neighboring nearest-neighbor vertices form elementary plaquettes, and hopping (including the nonlocal hybridization as well) is possible along the edges and diagonals of elementary plaquettes. On each vertex local onsite potentials are acting, and on each vertex local on-site Hubbard-type repulsion is present as interaction.

The remaining part of the paper is organized as follows. Section II presents the Hamiltonian, Sec. III describes an exact transformation of the Hamiltonian which allows the deduction of the presented results, Sec. IV analyzes the disorder present in the system and provides concrete examples for the emergence of the model conditions necessary for the solutions to occur, Sec. V presents the exact ground states, Sec. VI describes ground-state expectation values, and finally, Sec. VII concluding the paper closes the presentation.

\section{THE HAMILTONIAN OF THE MODEL}

The fixed topology of the described system allows us to treat the problem in a 2D tight-binding Hamiltonian defined in $\mathbf{r}$ space on a 2D Bravais lattice with disordered Hamiltonian parameters. For this system we consider a unit cell $I$ described by the primitive vectors $(\mathbf{x}, \mathbf{y})$, and we take into account two types of electrons denoted by the particle index $p$ as $p=d, f$. In these conditions our starting Hamiltonian has the form $\hat{H}=\hat{H}_{0}+\hat{H}_{i n t}$, where

$$
\begin{aligned}
& \hat{H}_{0}=\sum_{p=d, f} \sum_{p^{\prime}=d, f} \sum_{\sigma}\left[\sum_{\mathbf{r} \neq 0}\left(t_{\mathbf{i}, \mathbf{i}+\mathbf{r}, \mathbf{r}, \sigma}^{p, p^{\prime}} \hat{p}_{\mathbf{i}, \sigma}^{\dagger} \hat{p}_{\mathbf{i}+\mathbf{r}, \sigma}^{\prime}+\text { H.c. }\right)\right. \\
& \left.+t_{\mathbf{i}, \mathbf{i}, 0, \sigma}^{p, p^{\prime}} \hat{p}_{\mathbf{i}, \sigma}^{\dagger} \hat{p}_{\mathbf{i}, \sigma}^{\prime}\right], \\
& \hat{H}_{\text {int }}=\sum_{p=d, f} \sum_{\mathbf{i}} U_{\mathbf{i}}^{p} \hat{n}_{\mathbf{i}, \uparrow}^{p} \hat{n}_{\mathbf{i}, \downarrow}^{p} .
\end{aligned}
$$

In the one-particle part $\hat{H}_{0}$, the "length" of the hopping denoted by $\mathbf{r}$ with possible nonzero values $\mathbf{x}, \mathbf{y}, \mathbf{y}+\mathbf{x}, \mathbf{y}-\mathbf{x}$, is allowed to extend only to distances contained in $I$, i.e., nearest neighbors $(\mathbf{x}, \mathbf{y})$ and next-nearest neighbors $(\mathbf{y}+\mathbf{x}, \mathbf{y}-\mathbf{x})$ (see Fig. 1). Denoting by $N_{\Lambda}$ the number of lattice sites in the system, the random nature of $\hat{H}$ is given by (a) the $2 N_{\Lambda}$ independent, noncorrelated, random (repulsive) on-site Hubbard interactions $U_{\mathbf{i}}^{p}, p=d, f$ contained in $\hat{H}_{i n t}$, and (b) $2 N_{\Lambda}$ new independent, noncorrelated, random $\hat{H}_{0}$ parameters chosen (as will be clarified below) from the (site, direction, and spin dependent) $t_{\mathbf{i}, \mathbf{i}+\mathbf{r}, \mathbf{r}, \sigma}^{p, p^{\prime}}$ amplitudes. We underline that the $t^{p, p^{\prime}}$ coefficients contain hybridization $\left(p \neq p^{\prime}\right)$, and onsite potential $(\mathbf{r}=0)$ terms as well. 


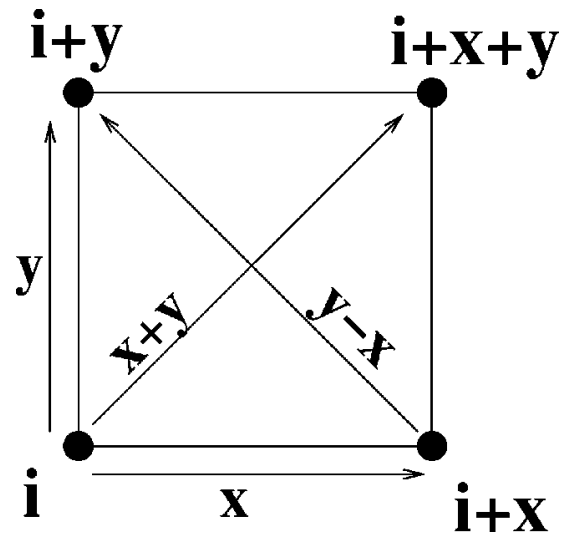

FIG. 1. Unit cell $I$ at site $\mathbf{i}$. The vectors $\mathbf{x}, \mathbf{y}$ denote the primitive vectors of the unit cell, and arrows indicate the possible $\mathbf{r}$ values allowed for the hoppings.

We demonstrate below that for certain local conditions imposed for $t^{p, p^{\prime}}$ parameters which maintain the number of $4 N_{\Lambda}$ independent noncorrelated random variables in the system, the exact multielectronic ground-state wave function of $\hat{H}$ in the interacting case can be explicitly given in an electron concentration dependent manner.

We mention that the spin-dependent nature of the $\hat{H}_{0}$ parameters is not essential for our deduction. The $\hat{H}_{0}$ parameters can be in principle spin dependent as well, and we underline this aspect, in order to extend the applicability of the results also to Hamiltonians with nondiagonal hoppings ${ }^{43,45-47}$ too. Furthermore, concerning the type of the model we use, we mention that for $U_{\mathbf{i}}^{d}=0$, the $\hat{H}$ from Eq. (1) represents a disordered periodic Anderson model (or Anderson lattice), while for $U_{\mathrm{i}}^{d} \neq 0$, Eq. (1) describes a disordered two-band Hubbard model ( $U_{\mathbf{i}}^{f} \neq 0$ always). Our results are applicable in both cases. For physical realization of such type of systems see, for example, Ref. 34.

We further consider that the mobility of the two types of electrons present in the system $(d$ and $f)$ is different, and the ratio in mobility is the same on all lattice sites. As a consequence, from the point of view of hopping amplitudes, starting from amplitudes written for $d$ electrons, we have

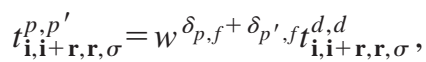

where $w$ is a (site independent) measure of the mobility ratios between $f$ and $d$ electrons. We mention that hopping amplitudes between different orbitals often satisfy such types of relations in real systems. ${ }^{45}$

Concerning again the $t^{p, p^{\prime}}$ terms, being interested in the behavior of particles given by the disordered hoppings and interactions, we only consider situations for which the localization of particles in local trapping centers is avoided, i.e., we have

$$
t_{\mathbf{i}, \mathbf{i}, 0, \sigma}^{p, p}>0 \text {. }
$$

a)

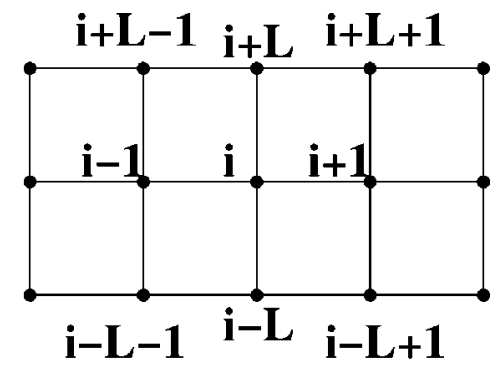

b)

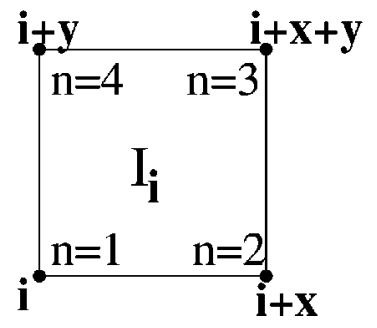

FIG. 2. (a) The numbering of sites in an $L \times L$ two-dimensional lattice around the lattice site $\mathbf{i}$, and (b) the unit cell $I_{\mathbf{i}}$ placed at an arbitrary lattice site $\mathbf{i}$, together with the $\mathbf{i}$ independent notation ( $n$ $=1,2,3,4)$ of sites inside $I_{\mathbf{i}}$. $(\mathbf{x}, \mathbf{y})$ are the primitive vectors of the unit cell.

In the following section we are presenting a transformation of $\hat{H}$ in a form that allows us to obtain exact ground states in its spectrum.

\section{THE TRANSFORMATION OF THE HAMILTONIAN}

Let us introduce a numbering of the lattice sites by the integer number $l$ in the studied 2D lattice containing $N_{\Lambda}$ $=L \times L$ lattice sites, starting from the down-left corner in the lowest row $(l=1)$, going from left to right up to the end of the first row $(l=L)$, then going upward and continuing with the second row again from left to right, and so on. In this manner, for example, around an arbitrary lattice site $\mathbf{i}$, numbered by $l=i$, we find the site numbering notations presented in Fig. 2(a). The introduced notation allows us to turn from a vectorial site notation to a scalar one, which simplifies as well the notation of the Hamiltonian parameters. For example, the $t_{\mathbf{i}, \mathbf{i}+\mathbf{r}, \mathbf{r}, \sigma}^{p, p^{\prime}}$ for $\mathbf{r}=\mathbf{x}(\mathbf{r}=\mathbf{y})$ at site $l=i$ becomes $t_{i, i+1, x, \sigma}^{p, p^{\prime}}\left(t_{i, i+L, y, \sigma}^{p, p^{\prime}}\right)$ [see Fig. 2(a)]. Similarly, the nextnearest-neighbor components $(\mathbf{x}+\mathbf{y}, \mathbf{y}-\mathbf{x})$ become $t_{i, i+1+L, x+y, \sigma}^{p, p^{\prime}}, t_{i+1, i+L, y-x, \sigma}^{p, p^{\prime}}$.

Let us further introduce a plaquette operator $\hat{A}_{\mathbf{i}, \sigma}$ defined for every arbitrary cell $I_{\mathrm{i}}$ taken at site $\mathbf{i}$ [see Fig. 2(b)]. The cell $I_{\mathbf{i}}$ is denoted by its down-left corner $\mathbf{i}$. The sites inside $I_{\mathbf{i}}$ are numbered in a cell independent manner by the index $n$ $=1,2,3,4$ starting from the site $\mathbf{i}$ and counting anticlockwise inside the unit cell $I_{\mathrm{i}}$ [see Fig. 2(b)]. In these conditions we obtain for $\hat{A}_{\mathbf{i}, \sigma}$ the expression

$$
\hat{A}_{\mathbf{i}, \sigma}=\sum_{n=1}^{4}\left(a_{n, d} \hat{d}_{\mathbf{i}+\mathbf{r}_{n}, \sigma}+a_{n, f} \hat{f}_{\mathbf{i}+\mathbf{r}_{n}, \sigma}\right),
$$


where $a_{n, p}$ are numerical coefficients, the same in all unit cells, and $\mathbf{r}_{1}=0, \mathbf{r}_{2}=\mathbf{x}, \mathbf{r}_{3}=\mathbf{x}+\mathbf{y}, \mathbf{r}_{4}=\mathbf{y}$ (detailed description of this procedure can be found in Ref. 40. For unit-cell independent notation of the coefficients $a_{n, p}$ see Ref. 42). Let us further connect to every unit cell $I_{l=i}$, two random variables $\epsilon_{i, \uparrow}$ and $\epsilon_{i, \downarrow}$.

Our results are based on the observation that if we define the plaquette operator parameters $a_{n, p}$ via the nonlinear system of equations

$$
\begin{gathered}
t_{i, i+1, x, \sigma}^{d, d}=a_{1, d}^{*} a_{2, d} \epsilon_{i, \sigma}+a_{4, d}^{*} a_{3, d} \epsilon_{i-L, \sigma}, \\
t_{i, i+L, y, \sigma}^{d, d}=a_{1, d}^{*} a_{4, d} \epsilon_{i, \sigma}+a_{2, d}^{*} a_{3, d} \epsilon_{i-1, \sigma}, \\
t_{i, i+1+L, x+y, \sigma}^{d, d}=a_{1, d}^{*} a_{3, d} \epsilon_{i, \sigma}, \\
t_{i+1, i+L, y-x, \sigma}^{d, d}=a_{2, d}^{*} a_{4, d} \epsilon_{i, \sigma}, \\
t_{i, i, 0, \sigma}^{d, d}=\left|a_{1, d}\right|^{2} \epsilon_{i, \sigma}+\left|a_{2, d}\right|^{2} \epsilon_{i-1, \sigma}+\left|a_{3, d}\right|^{2} \epsilon_{i-1-L, \sigma} \\
+\left|a_{4, d}\right|^{2} \epsilon_{i-L, \sigma},
\end{gathered}
$$

and $a_{n, f}=w a_{n, d}$ holds, where the parameter $w$ [see Eq. (2)] is real but arbitrary, then taking into account periodic boundary conditions, the one-particle part $\hat{H}_{0}$ of the starting Hamiltonian from Eq. (1) becomes

$$
\hat{H}_{0}=\sum_{\mathbf{i}, \sigma} \epsilon_{\mathbf{i}, \sigma} \hat{A}_{\mathbf{i}, \sigma}^{+} \hat{A}_{\mathbf{i}, \sigma} .
$$

Comparing the last equality of Eq. (5) to Eq. (3), we obtain the condition $\epsilon_{i, \sigma}>0$, although $\epsilon_{i, \sigma}$ are random variables. As a consequence, $\hat{H}$ in Eq. (1) becomes positive semidefinite:

$$
\hat{H}=\sum_{\mathbf{i}, \sigma} \epsilon_{\mathbf{i}, \sigma} \hat{A}_{\mathbf{i}, \sigma}^{+} \hat{A}_{\mathbf{i}, \sigma}+\sum_{p=d, f} \sum_{\mathbf{i}} U_{\mathbf{i}}^{p} \hat{n}_{\mathbf{i}, \uparrow}^{p} \hat{n}_{\mathbf{i}, \downarrow}^{p},
$$

and this property preserves the potential possibility to obtain the explicit form of the ground state in the interacting case.

\section{THE DISORDER IN THE SYSTEM}

\section{A. The presence of randomness in the model}

Before going further, we should analyze the kind of randomness we have in the system. We start with the observation that $\hat{H}$ in Eq. (7), which will be analyzed further on, is clearly disordered since it contains $4 N_{\Lambda}$ independent, noncorrelated (non-negative), arbitrary random variables $\epsilon_{i, \sigma}$ and $U_{i}^{p}$. However, the randomness must be understood not only at the level of the transformed Hamiltonian, Eq. (7), but also at the level of the starting $\hat{H}$ presented in Eq. (1). Since the disorder in $\hat{H}_{\text {int }}$ is the sames in Eqs. (1) and (7), this question relates to only the randomness in $\hat{H}_{0}$. In order to understand the source of the disorder in $\hat{H}_{0}$ from Eq. (1), we have two different alternatives.

One possibility for this is to observe the linear relationship between the on-site energy levels $t_{i, i, 0, \sigma}^{d, d}$ and $\epsilon_{i, \sigma}$ in the last row of Eq. (5). As a consequence, we can consider that the initial disordered parameters of the starting $\hat{H}_{0}$ in Eq. (1) are the $\eta_{i, \sigma}=t_{i, i, 0, \sigma}^{d, d}$ variables whose number is $2 N_{\Lambda}$, and the $\epsilon_{i, \sigma}$ new disordered parameters from Eq. (7) are obtained from these by a linear transformation

$$
\begin{gathered}
\eta_{i, \sigma}=\left|a_{1, d}\right|^{2} \epsilon_{i, \sigma}+\left|a_{2, d}\right|^{2} \epsilon_{i-1, \sigma}+\left|a_{3, d}\right|^{2} \epsilon_{i-1-L, \sigma} \\
+\left|a_{4, d}\right|^{2} \epsilon_{i-L, \sigma},
\end{gathered}
$$

which contains also $2 N_{\Lambda}$ equations. Since the $\eta_{i, \sigma}$ disordered parameters are independent, in this view one can consider that $\hat{H}$, besides the randomness in $\hat{H}_{\text {int }}$, possesses also "diagonal disorder" at the level of $\hat{H}_{0}$ in Eq. (1).

Alternatively, one can consider in Eq. (5) the unit-cell diagonal hopping amplitudes $\left(t_{i, i+L, y-x, \sigma}^{d, d}, t_{i, i+1+L, x+y, \sigma}^{d, d}\right)$ directly proportional to $\epsilon_{i, \sigma}$ as the source of the disorder in the one-particle part of the Hamiltonian, $\hat{H}_{0}$ in Eq. (1). In this case, $\hat{H}$ is considered to contain besides the randomness in $\hat{H}_{\text {int }}$ also "nondiagonal" disorder at the level $\hat{H}_{0}$.

In both cases, the remaining equalities in Eq. (5) must be considered as local constraints necessary for the solutions to occur. Since the number of $\hat{H}_{0}$ parameters in Eq. (1) is much higher than the number $2 N_{\Lambda}$ of random one-particle variables, these constraints do not alter the random nature of the disordered variables $\left(\left\{U_{i}^{p}, \eta_{i, \sigma}\right\}\right.$, or $\left.\left\{U_{i}^{p}, \epsilon_{i, \sigma}\right\}\right)$. Rather, they lead to (1) interdependences between $\hat{H}_{0}$ parameters not containing the disordered variables and (2) connect other $\hat{H}_{0}$ parameters to $\eta_{i, \sigma}$ or $\epsilon_{i, \sigma}$ disordered variables. These constraints emerge in the process of the transformation of Eq. (1) into Eq. (7), and we underline that our solutions are valid only in the case when this transformation can be done [i.e., Eq. (5) holds]. Both cases mentioned above as nondiagonal and diagonal disorders in $\hat{H}_{0}$ will be analyzed in detail below.

\section{B. Connections to the solutions obtained for Penrose tiling}

Considering the disorder in $\hat{H}_{0}$ as nondiagonal, the solutions presented here can be viewed as arising from extension of the conditions used in the exact study of the Penrose tiling. In order to understand this statement, let us introduce the constants $K_{1}=a_{1, d}^{*} a_{3, d}, K_{2}=a_{2, d}^{*} a_{4, d}$, and observe that since $\epsilon_{i, \sigma}$ are random, the diagonal (next-nearest neighbor) hopping matrix elements in every unit cell $I_{i}$, namely, $t_{i, \sigma}^{d,+}$ $=t_{i, i+1+L, x+y, \sigma}^{d, d}$ and $t_{i, \sigma}^{d,-}=t_{i+1, i+L, y-x, \sigma}^{d, d}$, excepting their ratio $\left(K_{1} / K_{2}\right)$, remain random as well

$$
t_{i, \sigma}^{d,+}=K_{1} \epsilon_{i, \sigma}, \quad t_{i, \sigma}^{d,-}=K_{2} \epsilon_{i, \sigma} .
$$

Considering for example the hopping amplitudes without directional dependence, i.e., $t_{i, 2, \sigma}^{d}=t_{i, \sigma}^{d, \pm}$, and taking for simplicity $K_{1}=K_{2}=1$, we obtain

$$
t_{i, 2, \sigma}^{d}=\epsilon_{i, \sigma},
$$

which (excepting the fixed sign of $\epsilon_{i, \sigma}>0$ ) means completely random and independent unit-cell diagonal hoppings for all spins in all unit cells (see Fig. 3). As a consequence, based on Eq. (9) or its particular form from Eq. (10), we see 


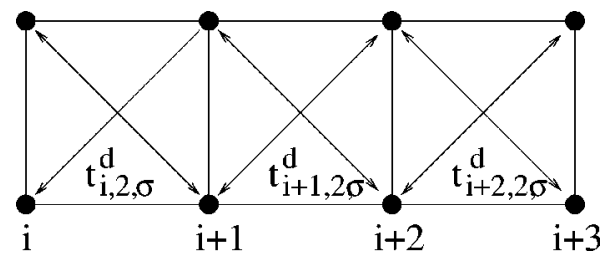

FIG. 3. The independent diagonal $t_{i, 2, \sigma}^{d}$ hopping amplitudes indicated by arrows in different unit cells.

that the randomness given by $\left\{U_{i}^{p}, \epsilon_{i, \sigma}\right\}$ in Eq. (7) can be considered to originate from the randomness given by $\left\{U_{i}^{p}, t_{i, \sigma}^{d, \pm}\right\}$ at the level of the starting $\hat{H}$ presented in Eq. (1). In this case, once the hopping amplitudes along the diagonals of every unit cell have been randomly chosen, the remaining $t^{p, p^{\prime}}$ parameters can be determined based on them. The study of Eq. (5) shows that fixing the $t_{i, \sigma}^{d, \pm}$ values, we have the liberty to choose independently two more constants relating the one-particle part of the Hamiltonian $\hat{H}_{0}$, namely, $K_{3}$ and $K_{4}\left(\operatorname{sgn} \bar{K}>0, \bar{K}=K_{2} K_{3} K_{4}\right)$ based on which

$$
\begin{array}{r}
t_{i, i+1, x, \sigma}^{d, d}=K_{3} t_{i-L+1, i, y-x, \sigma}^{d, d}+\frac{1}{K_{3}} t_{i, i+1+L, x+y, \sigma}^{d, d}, \\
t_{i, i+L, y, \sigma}^{d, d}=K_{4} t_{i, i+L-1, y-x, \sigma}^{d, d}+\frac{1}{K_{4}} t_{i, i+1+L, x+y, \sigma}^{d, d}, \\
t_{i, i, 0, \sigma}^{d, d}=\frac{K_{1}}{\bar{K}} t_{i, i+L+1, x+y, \sigma}^{d, d}+\frac{\bar{K}}{K_{1}} t_{i-L-1, i, x+y, \sigma}^{d, d} \\
+\frac{K_{4}}{K_{3}} t_{i, i+L-1, y-x, \sigma}^{d, d}+\frac{K_{3}}{K_{4}} t_{i-L+1, i, y-x, \sigma}^{d, d},
\end{array}
$$

and the numerical coefficients present in Eq. (5) in function of $K_{m}(m=1,2,3,4)$, arbitrary parameters become $a_{1, d}^{*} a_{3, d}$ $=K_{1}, \quad a_{2, d}^{*} a_{4, d}=K_{2}, \quad a_{1, d}^{*} a_{2, d}=K_{1} / K_{3}, \quad a_{1, d}^{*} a_{4, d}=K_{1} / K_{4}$, $a_{2, d}^{*} a_{3, d}=K_{2} K_{4}, \quad a_{4, d}^{*} a_{3, d}=K_{2} K_{3}, \quad\left|a_{1, d}\right|^{2}=K_{1}^{2} / \bar{K}, \quad\left|a_{2, d}\right|^{2}$ $=K_{2} K_{4} / K_{3},\left|a_{3, d}\right|^{2}=\bar{K}$, and $\left|a_{4, d}\right|^{2}=K_{2} K_{3} / K_{4}$. In order to have real value for all $t^{p, p^{\prime}}$ parameters, all $K_{m}$ must be real. To understand in detail Eq. (11), let us introduce short notations as well for nearest neighbor and local amplitudes in the form $t_{i, \sigma}^{d, x}=t_{i, i+1, x, \sigma}^{d, d}, \quad t_{i, \sigma}^{d, y}=t_{i, i+L, y, \sigma}^{d, d}, \quad t_{i, \sigma}^{d, 0}=t_{i, i, 0, \sigma}^{d, d}$, which represent the $t_{i, \sigma}^{d, \nu}$ amplitudes for $d$ electrons with spin $\sigma$ in unit cell $I_{i}$ for $\nu=|\mathbf{r}|$. Using these notations, Eq. (11) becomes

$$
\begin{gathered}
t_{i, \sigma}^{d, x}=K_{3} t_{i-L, \sigma}^{d,-}+K_{3}^{-1} t_{i, \sigma}^{d,+}, \quad t_{i, \sigma}^{d, y}=K_{4} t_{i-1, \sigma}^{d,-}+K_{4}^{-1} t_{i, \sigma}^{d,+}, \\
t_{i, \sigma}^{d, 0}=R_{1} t_{i, \sigma}^{d,+}+R_{1}^{-1} t_{i-L-1, \sigma}^{d,+}+R_{2} t_{i-1, \sigma}^{d,-}+R_{2}^{-1} t_{i-L, \sigma}^{d,-}
\end{gathered}
$$

where $R_{1}=K_{1} / \bar{K}$ and $R_{2}=K_{4} / K_{3}$. As shown in Fig. 4, the $t_{i, \sigma}^{d, \nu}$ amplitudes presented in Eq. (12) are determined by the $t^{d, \pm}$ unit-cell diagonal amplitudes that surround $t_{i, \sigma}^{d, \nu}$. For example, as seen from Fig. 4(a), the $t_{i, \sigma}^{d, x}$ nearest-neighbor hopping amplitude (full line arrow) is given by the $t_{i, \sigma}^{d,+}$ and $t_{i-L, \sigma}^{d,-}$ random unit-cell diagonal amplitudes (dotted line ar- a)

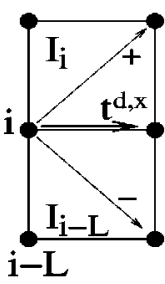

b)

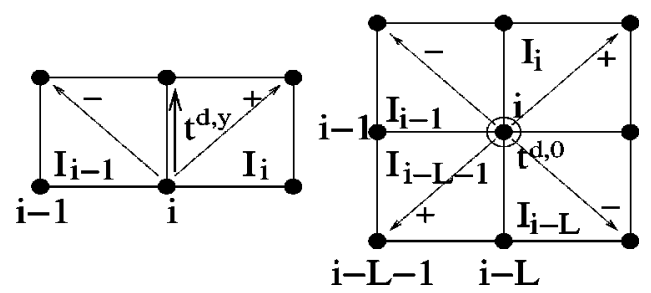

FIG. 4. The $t_{i, \sigma}^{d, \nu}$ amplitudes at site $i$ for (a) $\nu=x$, (b) $\nu=y$, and (c) $\nu=0$, respectively. $t_{i, \sigma}^{d, \nu}$ is denoted by full arrow in (a), (b), and by a circle in (c). Dotted arrows with \pm label indicate the $t_{j, \sigma}^{d, \pm}$ random amplitudes that enter in the expression of $t_{i, \sigma}^{d, \nu}$ presented in the plots (a), (b), (c) from Eq. (12). In all plots the notation of the unit cell $I_{j}$ at site $j$ containing $t_{j, \sigma}^{d, \nu}$ is also presented. For example, in (a) $I_{i}$ defined at site $i$ contains $t_{i, \sigma}^{d,+}$, and $I_{i-L}$ defined at the site $i$ $-L$ contains $t_{i-L, \sigma}^{d,-}$.

rows) which start from the same site $i$ and intercalate $t_{i, \sigma}^{d, x}$. Similar situation is present for $t_{i, \sigma}^{d, y}$ in $y$ direction [see Fig. 4(b)], while $t_{i, \sigma}^{d, 0}$ as seen in Fig. 4(c) is determined by the four $t^{d, \pm}$ "plaquette-diagonal" amplitudes that start from the same site $i$.

Concerning Eq. (12), we mention that in the study of disordered systems, constraints (correlations) between bond and site properties are often considered. The constraints a priori introduced can be in some cases even of long-range type, ${ }^{52}$ as taken, for example, in the case of isotropically correlated random potentials, ${ }^{48}$ correlated networks, ${ }^{49}$ etc., and even calculation techniques have been developed in order to deal with "constrainted" disorder, for example, in the form of correlated random numbers, ${ }^{50}$ or random matrices with symmetry properties or holding constraints. ${ }^{49}$ In our case, local constraints exist which connect the plaquette-diagonal bond hoppings (considered as the true independent random variables of $\hat{H}_{0}$ ) to edge (nearest-neighbor) bond hoppings and local one-particle potentials. Since the plaquette-diagonal bond can be unambiguously connected to the plaquette, in the described case, random plaquette properties (i.e., random bonds connected to plaquettes), through local constraints presented in Eq. (12), fix nearest-neighbor or local amplitudes.

Concrete physical situations where in disordered systems the random plaquette properties determine nearest-neighbor or local amplitudes are also known in the literature. For example, in the case of topologically disordered system of Caer type $^{44}$ using random mosaics, very similar to Voronoi tessellation generated from disordered arrangement of particles, ${ }^{51}$ random flips of plaquette-diagonal bonds performed with a given probability determine the local nearest-neighbor hoppings, and introduce in this way the disorder in the system. ${ }^{18}$ Concerning disordered on-site one-particle terms generated by random bonds connected to plaquette properties, we mention, for example, the Penrose lattice ${ }^{22,23}$ case, where the on-site one-particle potentials have been introduced by the local coordination number. ${ }^{24}$ In the mentioned case, practically the random on-site one-particle potential at site $i$ is determined by the number of bonds entering in the site $i$. Our on-site potential $t_{i, i, 0, \sigma}^{p, p}$ given in Eq. (12) and presented in 
Fig. 4(c) has clear similarities with this choice, since it reduces to a such type of behavior in the case in which all $t_{i, \sigma}^{d, \pm}$ unit-cell diagonal hoppings are equal, and, for example, for all $m, K_{m}=1$. The difference between Eq. (12) and Ref. 24 from the point of view of the random on-site potential is that in our case, the on-site potential is determined by the value of the random bonds entering in the site, while in Ref. 24, by the number of the bonds entering in the site. So contrary to Refs. 24-26, where the study has been concentrated on the effects of the lattice topology alone, in this paper we analyze the problem in a fixed topology, concentrating on random $t^{p, p^{\prime}}$ values. We further mention that in the Penrose lattice case, when also the plaquette-diagonal hopping amplitudes are taken into account at the level of exact independent electron eigenstates, ${ }^{27}$ solutions are found only when constraints are present between hopping amplitudes.

Let us consider a concrete physical example in support of Eq. (12) which demonstrates as well that solutions deduced in the context of Penrose lattice ${ }^{27}$ use quite similar conditions. For this, let us take a simple spin-independent case $t_{i, j, r, \sigma}^{p, p^{\prime}}=t_{i, j, r,-\sigma}^{p, p^{\prime}}=t_{i, j, r}^{p, p^{\prime}}$, and consider a situation for which randomly positioned $A$ or $B$ atoms in the middle of the elementary plaquettes providing the random unit-cell diagonal hoppings $t_{i, \sigma}^{d, \pm}=t_{i}^{d, \pm}$ introduce the randomness in $\hat{H}_{0}$. In this situation, $t_{i}^{d, \pm}$ is either $t^{d, \pm}(A)$ or $t^{d, \pm}(B)$, depending on the type of atom situated in the middle of a unit-cell. For this example Eq. (12) expresses the fact that the hopping amplitude along a bond (nearest-neighbor hoppings $t_{i}^{d, x}$ and $t_{i}^{d, y}$ ) depends on the randomly situated atoms $A$ and $B$ placed in the neighborhood of the bond, and that the on-site energy of a given site $\left(t_{i}^{d, 0}\right)$ depends on the randomly positioned $A$ and $B$ atoms in the neighborhood of the site, which are physically quite acceptable conditions. The linearity of these interdependences can be physically motivated by the small influence of the atoms $A$ or $B$ not situated directly on the bond or on the site. For the presented example, $t^{d, \pm}(A),\left[t^{d, \pm}(B)\right]$ in the Penrose lattice case would correspond to the notations $d_{1}, d_{2},\left(d_{3}, d_{4}\right)$ used in Ref. 27. Furthermore, in Ref. 27 $t_{i}^{d, x}=t_{i}^{d, y}=1$ is considered, and our $t_{i}^{d, 0}$ is denoted by $\epsilon_{i}$. The conditions in which solutions are obtained for the Penrose lattice case [see Eqs. (3.9), (3.11), and (3.13) in Ref. 27] are in fact linear relations of the type of our Eq. (12). The main difference between our model and that of Ref. 27 at the level of $\hat{H}_{0}$ is that in our case, the plaquettes described by $t^{d, \pm}(A)$ and $t^{d,{ }^{ \pm}}(B)$ can emerge completely random, while in Ref. 27 the plaquettes (rhombi) described by $\left(d_{1}, d_{2}\right)$ and $\left(d_{3}, d_{4}\right)$ emerge only with the randomness allowed by the Penrose tiling. Because of this reason, our solutions extend the exact solution possibilities known in Penrose lattice case to nonquasicrystalline disordered systems even in the presence of electron-electron interaction.

\section{The disorder seen in the one-particle part of $\hat{\boldsymbol{H}}$ as diagonal disorder}

Considering the source of the disorder in $\hat{H}_{0}$ diagonal, the random parameters of the model become $U_{i}^{p}$ and $\eta_{i, \sigma}$. In this case Eq. (5) requires two supplementary conditions to be satisfied as follows.
(1) The next-nearest-neighbor hoppings surrounding a nearest-neighbor hopping [see Figs. 4(a) and 4(b), and first two equalities from Eq. (12)] must be related at the level of hopping amplitudes. These conditions are not specific for the presented disordered model, but are rather connected to the method itself. Indeed, in such conditions we find solving nondisordered cases as well (see Refs. 39-42), and the obtained hopping amplitude ratios are delimitating parameter space regions where the obtained solutions are valid.

(2) The next-nearest-neighbor (unit-cell diagonal) hoppings starting from a given site $i$ are all together linearly related to the on-site energy level (considered disordered here) at the site $i$ [see the last equality from Eq. (12), or alternatively Eq. (8), and Fig. 4(c)]. This local constraint, in this form, is specific for the random case studied here.

Even if the conditions (1) and (2) presented above seem to be quite specific at first view, we show below that they are compatible with the presence of the diagonal disorder on physical grounds. For this to be visible, we analyze a simple pedagogical spin-independent hopping case which is $x-y$ symmetric as well, so $t_{i, \sigma}^{d, x}=t_{i, \sigma}^{d, y}=t_{i, \sigma}^{d, \nu=1}=t_{i}^{d, 1}, t_{i, \sigma}^{d, \pm}=t_{i, 2, \sigma}^{d}$ $=t_{i, \sigma}^{d, \nu}=2=t_{i}^{d, 2}$, and $K_{3}=K_{4}=K_{0}, R_{2}=1$ [see Eq. (12)]. Let us further consider for the study that the random on-site potential $\eta_{i}$ is created by the randomly positioned $A_{\tau}$ atom at site $i$ of the lattice with lattice spacing $a$, where the index $\tau$ fixes the type of the atom. In this manner, if the atom $A_{\tau}$ will be placed at site $i$, it creates the on-site energy level $\eta_{i}$ $=\eta_{\tau}$. After this step, we must model the expression of the distance dependent hopping amplitude $t_{i}(r)$ for the electron which starts the hopping from $i$. Taking into account a simple exponential distance decrease, we may simply take $t_{i}(r)$ $=C_{i} B_{i}(A v) e^{-\alpha r}$, where the constant $\alpha$ describes the distance decrease $(r \neq 0$ is considered). The amplitude $C_{i} B_{i}(A v)$ is built up from the component $C_{i}=C_{\tau}$ depending on the energy level at site $i$ (the atom $A_{\tau}$ present at site $i$ ), and the average effect of all surrounding atoms felt at site $i$ denoted by $B_{i}(A v)$. Since only $t_{i}^{d, 1}$ and $t_{i}^{d, 2}$ hoppings are considered, we have for the $t_{i}^{d, 1}\left(t_{i}^{d, 2}\right)$ case the $r=a(r$ $=a \sqrt{2})$ argument value in $t_{i}(r)$.

After these considerations, the conditions (1) and (2) mentioned above look as follows. Condition (1) links together three hopping amplitudes for hoppings which start from the same site $i$ [see Figs. 4(a) and 4(b), and the equalities relating $t_{i}^{d, 1}$ from Eq. (12)], providing the condition $e^{\alpha a(\sqrt{2}-1)}=K_{0}+\left(1 / K_{0}\right)$. As can be seen, condition (1) determines in fact the strength of the hopping (parameter $\alpha$ ) through the constant $K_{0}$, and is not specific for the random case, as mentioned above. Rather, it fixes the $t^{d, 1} / t^{d, 2}$ ratio introducing limits for the validity of the solutions in the $T$ $=0$ phase diagram of the starting Hamiltonian. As a consequence, we can further analyze condition (2) considering $\alpha$ a known parameter.

Condition (2) [Fig. 4(c), and the equality relating $\eta_{i}$ $=t_{i, \sigma}^{d, 0}$ from Eq. (12)] links together four next-nearestneighbor hoppings which again start from the same site. As a consequence, taking into account that the atom $A_{\tau}$ is placed on the lattice site $i$, we find $\eta_{\tau}=C_{\tau} B_{i}(A v)\left[2+R_{1}\right.$ $\left.+\left(1 / R_{1}\right)\right] e^{-\sqrt{2} \alpha a}$. The remaining $B_{i}(A v)$ coefficients must 
be deduced at each site from the condition $t_{i, j}^{*}=t_{j, i}$. As can be seen, condition (2) through the parameter $R_{1}$ relates the disordered on-site energy level values to the hopping amplitude components $C_{i}$.

As presented above, the conditions necessary to be fulfilled for the solutions to emerge are present in disordered systems, being compatible to a truly acceptable physical background. Taking into account more complicated parametrizations for $t_{i}(r)$, the equation of $\eta_{i, \sigma}=t_{i, \sigma}^{d, 0}$ in Eq. (12) (since it has in its right side the same $r$ value) reduces to an equation for the amplitudes of the $r$ function in $t_{i}(r)$, while the remaining equalities in Eq. (12) determine the $t_{i}(a) / t_{i}(a \sqrt{2})$ ratios.

\section{THE GROUND-STATE WAVE FUNCTION}

Starting from the positive semidefinite structure of $\hat{H}$ in Eq. (7), the ground-state wave function $\left|\Psi_{g}\right\rangle$ is obtained for $\hat{H}\left|\Psi_{g}\right\rangle=0$. Now, let us concentrate first on the $\hat{H}_{0}$ component of $\hat{H}$ presented in Eq. (6). Taking into account Eq. (4), and as shown in Eq. (5), $a_{n, f}=w a_{n, d}$, where $w$ is arbitrary but real, we realize that

$$
\hat{A}_{\mathbf{i}, \sigma}=\sum_{n=1}^{4} a_{n, d}\left(\hat{d}_{\mathbf{i}+\mathbf{r}_{n}, \sigma}+w \hat{f}_{\mathbf{i}+\mathbf{r}_{n}, \sigma}\right),
$$

so in the right side of $\hat{H}_{0}$ in Eq. (6) only operators of the form $\hat{O}_{j, \sigma}=\left(\hat{d}_{j, \sigma}+w \hat{f}_{j, \sigma}\right)$ are present. If now we define

$$
\hat{\bar{O}}_{j, \sigma}^{\dagger}=\hat{d}_{j, \sigma}^{\dagger}-\frac{1}{w} \hat{f}_{j, \sigma}^{\dagger},
$$

which satisfies $\hat{O}_{j, \sigma} \hat{\bar{O}}_{j^{\prime}, \sigma^{\prime}}^{\dagger}=-\hat{\bar{O}}_{j^{\prime}, \sigma^{\prime}}^{\dagger} \hat{O}_{j, \sigma}$, then taking $|\Psi\rangle$ $=\Pi_{j}\left[\hat{\bar{O}}_{j, \sigma}^{\dagger}+v_{j} \hat{\bar{O}}_{j,-\sigma}^{\dagger}\right]|0\rangle$, where $\Pi_{j}$ is taken over different (although arbitrary) lattice sites, $v_{i}$ are arbitrary coefficients, and $|0\rangle$ is the bare vacuum with no fermions present, we obtain

$$
\sum_{\mathbf{i}, \sigma} \epsilon_{\mathbf{i}, \sigma} \hat{A}_{\mathbf{i}, \sigma}^{+} \hat{A}_{\mathbf{i}, \sigma}|\Psi\rangle=0
$$

Since $|\Psi\rangle$ introduces fermions ( $d$ or $f$ ) with arbitrary spin, strictly on different sites, double occupancy is avoided, and

$$
\sum_{p=d, f} \sum_{\mathbf{i}} U_{\mathbf{i}}^{p} \hat{n}_{\mathbf{i}, \uparrow}^{p} \hat{n}_{\mathbf{i}, \downarrow}^{p}|\Psi\rangle=0
$$

holds as well. Since the minimum possible eigenvalue of $\hat{H}$ in Eq. (7) is zero, the ground state for arbitrary $N \leqslant N_{\Lambda}$, where $N$ represents the number of electrons within the system, becomes

$$
\left|\Psi_{g}\right\rangle=\prod_{j}^{N}\left[\hat{\bar{O}}_{j, \sigma}^{\dagger}+v_{j} \hat{\bar{O}}_{j,-\sigma}^{\dagger}\right]|0\rangle .
$$

In Eq. (17), the $\Pi_{j}^{N}$ product must be taken over $j$ sites which can be arbitrarily chosen, and different $j$ values must be related to strictly different lattice sites. The ground-state wave function of $\hat{H}$ given in Eq. (7) for $N \leqslant N_{\Lambda}$ (i.e., at and below quarter filling) can be always written in the form of Eq. (17). As a consequence, for $N=N_{\Lambda}$ the ground state becomes

$$
\left|\Psi_{g}\left(N=N_{\Lambda}\right)\right\rangle=\prod_{j=1}^{N_{\Lambda}}\left[\hat{\bar{O}}_{j, \sigma}^{\dagger}+v_{j} \hat{\bar{O}}_{j,-\sigma}^{\dagger}\right]|0\rangle .
$$

For $N<N_{\Lambda}$, since the $j$ sites in Eq. (17) can be arbitrarily chosen, the complete ground state becomes

$$
\left|\Psi_{g}\left(N<N_{\Lambda}\right)\right\rangle=\sum_{R_{N}}\left\{\alpha_{R_{N_{j}}} \prod_{R_{N}}\left[\hat{\bar{O}}_{j, \sigma}^{\dagger}+v_{j} \hat{\bar{O}}_{j,-\sigma}^{\dagger}\right]\right\}|0\rangle,
$$

where the sum $\Sigma_{R_{N}}$ is made over all different $R_{N}$ domains containing $N<N_{\Lambda}$ lattice sites from the system, and $\alpha_{R_{N}}$ are numerical coefficients. Furthermore, it is important to underline that Eqs. (18) and (19) represent the ground state only in the interacting case [at least one of on-site two-particle interactions $U_{i}^{p}$ must be nonzero at all sites $i$, since otherwise, because of the presence of the double occupancy, (e.g., $\hat{\bar{O}}_{j, \sigma}^{\dagger} \hat{\bar{O}}_{j,-\sigma}^{\dagger}$ products in the ground state wave function), the expression of $\left|\Psi_{g}\right\rangle$ in Eq. (17) is no more valid].

In my knowledge, Eqs. (18) and (19) contain the first exact multielectronic ground-state wave functions obtained in 2D for a disordered system in the interacting case. As explained above, Eqs. (18) and (19) are no more eigenstates for the independent electron approximation, i.e., $U_{\mathbf{i}}^{d}=U_{\mathbf{i}}^{f}$ $=0$. Since the ground state in the interacting case, even for infinitesimal interaction, changes qualitatively in comparison to the noninteracting case, Eqs. (18) and (19) cannot be connected in a perturbative way to the ground state of the disordered but noninteracting system.

The ground states (GS's) presented above are strongly degenerated. Their degeneracy at quarter filling is given by the $N$ arbitrary $v_{i}$ values, and the arbitrary (but nonzero) $w$, is proportional to the size of the system. The existence of such type of states for 2D Penrose-type lattices has been first conjectured by Semba and Ninomiya ${ }^{53}$ and Kohmoto and Sutherland, ${ }^{24}$ and further analyzed in Refs. 26 and 54. From the reported results here it can be seen that this property is present also for other systems as well in the multielectronic and interacting case too, at least for $N=N_{\Lambda}$. We stress however that in the case $N<N_{\Lambda}$, the degree of the degeneracy strongly increases given as well by the geometrical degeneracy present in Eq. (19). The order of magnitude of the degeneration becomes in this case $N_{R}=N_{\Lambda}$ !/ $\left[N !\left(N_{\Lambda}-N\right) !\right]$.

\section{GROUND-STATE EXPECTATION VALUES}

\section{A. The localized case}

Despite the possibility to chose the Hamiltonian parameters in a spin-dependent way, the obtained GS is globally paramagnetic. At $1 / 4$ filling $\left(N=N_{\Lambda}\right)$, the GS contains rigorously one electron on each site, so the hopping is completely forbidden in GS, and as a consequence, the system is localized, holding long-range density-density correlations. 
Indeed, calculating the ground-state expectation values through Eq. (18), in this case we find for arbitrary $i \neq j$ and all $\sigma, \sigma^{\prime}$

$$
\begin{aligned}
& \left\langle\hat{d}_{i, \sigma}^{\dagger} \hat{d}_{j, \sigma^{\prime}}\right\rangle=0, \quad\left\langle\hat{f}_{i, \sigma}^{\dagger} \hat{f}_{j, \sigma^{\prime}}\right\rangle=0, \\
& \left\langle\hat{d}_{i, \sigma}^{\dagger} \hat{f}_{j, \sigma^{\prime}}\right\rangle=0, \quad\left\langle\hat{f}_{i, \sigma}^{\dagger} \hat{d}_{j, \sigma^{\prime}}\right\rangle=0,
\end{aligned}
$$

where $\langle\cdots\rangle=\left\langle\Psi_{g}\left(N_{\Lambda}\right)|\cdots| \Psi_{g}\left(N_{\Lambda}\right)\right\rangle /\left\langle\Psi_{g}\left(N_{\Lambda}\right) \mid \Psi_{g}\left(N_{\Lambda}\right)\right\rangle$, $\left|\Psi_{g}\left(N_{\Lambda}\right)\right\rangle$ is presented in Eq. (18), and $\left\langle\Psi_{g}\left(N_{\Lambda}\right) \mid \Psi_{g}\left(N_{\Lambda}\right)\right\rangle$ $=\prod_{i=1}^{N_{\Lambda}}\left[\left(1+|w|^{-2}\right)\left(1+\left|v_{i}\right|^{2}\right)\right]$. The reason for Eq. (20) is simple: $\left|\Psi_{g}\left(N_{\Lambda}\right)\right\rangle$ contains exactly one electron on each lattice site, so $\left|\Psi_{1}\left(p, p^{\prime}\right)\right\rangle=\hat{p}_{i, \sigma}^{\dagger} \hat{p}_{j, \sigma^{\prime}}^{\prime}\left|\Psi_{g}\left(N_{\Lambda}\right)\right\rangle$, where $p, p^{\prime}$ $=d, f$ and $i \neq j$ contain a double occupancy, and as a consequence $\left|\Psi_{1}\left(p, p^{\prime}\right)\right\rangle$ and $\left|\Psi_{g}\left(N_{\Lambda}\right)\right\rangle$ are orthogonal. We underline that since Eq. (20) holds for arbitrary $v_{i}$, it remains the same after the average over the disorder ( $v_{i}$ variables). Denoting the translational invariant averages by $\langle\langle\cdots\rangle\rangle$ $=\int P\left(\left\{v_{i}\right\}\right)\left(\Pi_{i} d v_{i}\right)\langle\cdots\rangle$, where $P\left(\left\{v_{i}\right\}\right)$ describes the distribution of the disordered variables (being arbitrary here) and $\int P\left(\left\{v_{i}\right\}\right)\left(\Pi_{i} d v_{i}\right)=1$ holds by definition, Eq. (20) automatically implies as well $\left\langle\left\langle\hat{p}_{i, \sigma}^{\dagger} \hat{p}_{j, \sigma^{\prime}}^{\prime}\right\rangle\right\rangle=0$ for all $p, p^{\prime}$ $=d, f$, all $\sigma, \sigma^{\prime}$, and all $i \neq j$.

Furthermore, introducing for $i \neq j$ the notation $D(i, j)$ $=\left[\left(1+|w|^{-2}\right)\left(1+\left|v_{i}\right|^{2}\right)\left(1+\left|v_{j}\right|^{2}\right)\right]^{2}$, we find

$$
\begin{gathered}
\left\langle\hat{n}_{i, \sigma}^{d} \hat{n}_{j, \sigma}^{d}\right\rangle=D(i, j),\left\langle\hat{n}_{i,-\sigma}^{d} \hat{n}_{j,-\sigma}^{d}\right\rangle=\left|v_{i}\right|^{2}\left|v_{j}\right|^{2} D(i, j), \\
\left\langle\hat{n}_{i, \sigma}^{f} \hat{n}_{j, \sigma}^{f}\right\rangle=|w|^{-4} D(i, j), \\
\left\langle\hat{n}_{i,-\sigma}^{f} \hat{n}_{j,-\sigma}^{f}\right\rangle=\frac{\left|v_{i}\right|^{2}\left|v_{j}\right|^{2}}{|w|^{4}} D(i, j), \\
\left\langle\hat{n}_{i, \sigma}^{d} \hat{n}_{j, \sigma}^{f}\right\rangle=|w|^{-2} D(i, j), \quad\left\langle\hat{n}_{i, \sigma}^{d} \hat{n}_{j,-\sigma}^{f}\right\rangle=\frac{\left|v_{j}\right|^{2}}{|w|^{2}} D(i, j), \\
\left\langle\hat{n}_{i,-\sigma}^{d} \hat{n}_{j,-\sigma}^{f}\right\rangle=\frac{\left|v_{i}\right|^{2}\left|v_{j}\right|^{2}}{|w|^{2}} D(i, j), \\
\left\langle\hat{n}_{i,-\sigma}^{d} \hat{n}_{j, \sigma}^{f}\right\rangle=\frac{\left|v_{i}\right|^{2}}{|w|^{2}} D(i, j) .
\end{gathered}
$$

Starting from Eq. (21), for $\hat{n}_{i}=\Sigma_{\sigma}\left(\hat{n}_{i, \sigma}^{d}+\hat{n}_{i, \sigma}^{f}\right)$, based on Eq. (21) one obtains

$$
\left\langle\hat{n}_{i} \hat{n}_{j}\right\rangle=1,\left\langle\left\langle\hat{n}_{i} \hat{n}_{j}\right\rangle\right\rangle=1,
$$

where the second equality holds as explained below Eq. (20). Introducing now $\hat{S}_{i}^{z}=(1 / 2)\left[\left(\hat{n}_{i, \uparrow}^{d}+\hat{n}_{i, \uparrow}^{f}\right)-\left(\hat{n}_{i, \downarrow}^{d}+\hat{n}_{i, \downarrow}^{f}\right)\right]$, based again on Eq. (21), for $i \neq j$ we have

$$
\left\langle\hat{S}_{i}^{z} \hat{S}_{j}^{z}\right\rangle=\frac{p_{i} p_{j}}{4},
$$

where $p_{n}=\left(1-\left|v_{n}\right|^{2}\right) /\left(1+\left|v_{n}\right|^{2}\right)$ takes arbitrary values in the domain $(-1,+1)$, so $\left\langle\left\langle\hat{S}_{i}^{z} \hat{S}_{j}^{z}\right\rangle\right\rangle=0$ arises. As can be ob- served, $\left|\Psi_{g}\left(N_{\Lambda}\right)\right\rangle$ indeed describes a paramagnetic, completely localized ground state containing long-range densitydensity correlations. At quarter filling, since $\left|\Psi_{g}\left(N=N_{\Lambda}\right)\right\rangle$ coherently controls the particle number occupancy at all lattice sites forbidding the hopping (and nonlocal hybridization) in the same time, the GS clearly presents phase coherence over the whole lattice.

\section{B. The delocalized case}

Under quarter filling, empty sites emerge in the GS, Eq. (20) deduced through Eq. (19) does not hold, hopping is no more forbidden, and as a consequence, a delocalization occurs, the system becoming itinerant (remaining further paramagnetic). Indeed, in this case, at $N<N_{\Lambda}$, based on Eq. (19), the GS wave function can be written as $\left|\Psi_{g}(N)\right\rangle=\Sigma_{R_{N}} \alpha_{R_{N}}\left|\Psi\left(R_{N}\right)\right\rangle$, where $\left|\Psi\left(R_{N}\right)\right\rangle \equiv\left|\Psi_{R_{N}}\left(\left\{v_{i}\right\}\right)\right\rangle$ $=\sqrt{D_{R_{N}}^{-1}\left(\left\{v_{i}\right\}\right)} \Pi_{j \in R_{N}}\left(\hat{\bar{O}}_{j, \sigma}^{\dagger}+v_{j} \hat{\bar{O}}_{j,-\sigma}^{\dagger}\right)|0\rangle$ build up an orthonormalized wave-function set containing $N_{R}$ components, and we have $D_{R_{N}}\left(\left\{v_{i}\right\}\right)=\left(1+|w|^{-2}\right)^{N} \Pi_{j \in R_{N}}\left(1+\left|v_{j}\right|^{2}\right)$, and $\alpha_{R_{N}}$ are coefficients independent of the disordered $\left\{v_{i}\right\}$ set. The operators of the type $\hat{p}_{i, \sigma}^{\dagger} \hat{p}_{j, \sigma}^{\prime}$, where $p, p^{\prime}=d, f$, now have nonzero matrix elements between ground-state components $\left|\Psi\left(R_{N}\right)\right\rangle,\left|\Psi\left(R_{N}^{\prime}\right)\right\rangle$ describing $R_{N}, R_{N}^{\prime}$ domains of the form $R_{N}=D_{N-1}+\mathbf{i}, R_{N}^{\prime}=D_{N-1}+\mathbf{j}$, where $D_{N-1}$ represents an arbitrary region of the lattice containing $N-1$ lattice sites, and $\mathbf{i}, \mathbf{j}$ are representing two different but arbitrary (not necessarily nearest-neighbor) sites of the lattice. We have, for example,

$$
\begin{aligned}
& \left\langle\Psi\left(R_{N}\right)\left|\hat{d}_{i, \sigma}^{\dagger} \hat{d}_{j, \sigma}\right| \Psi\left(R_{N}^{\prime}\right)\right\rangle=\frac{\left(1+|w|^{-2}\right)^{-1}}{\sqrt{\left(1+\left|v_{i}\right|^{2}\right)\left(1+\left|v_{j}\right|^{2}\right)}}, \\
& \left\langle\Psi\left(R_{N}\right)\left|\hat{f}_{i, \sigma}^{\dagger} \hat{f}_{j, \sigma}\right| \Psi\left(R_{N}^{\prime}\right)\right\rangle=\frac{\left(1+|w|^{2}\right)^{-1}}{\sqrt{\left(1+\left|v_{i}\right|^{2}\right)\left(1+\left|v_{j}\right|^{2}\right)}} .
\end{aligned}
$$

Since the disordered variables emerge in Eq. (24) through $\left|v_{i}\right|,\left|v_{j}\right|$ non-negative numbers, the average over the disorder maintains the nonzero values in Eq. (24). As a consequence, the hopping being no more forbidden, the system becomes indeed itinerant. Since as seen from Eq. (24) all $d$ or $f$ electrons can hop everywhere in the ground state, the wave function in Eq. (19) is clearly an extended state. The conducting nature of the extended states can be demonstrated (see, for example, Ref. 55) through the variation of the chemical potential as the number of electrons vary. For this reason, let us observe that the ground-state wave function from Eq. (19) acting on the Hamiltonian from Eq. (7), by the construction of the wave function as explained in Sec. V, has the property $\hat{H}\left|\Psi_{g}\left(N<N_{\Lambda}\right)\right\rangle=E_{g}(N)\left|\Psi_{g}\left(N<N_{\Lambda}\right)\right\rangle=0$, where $E_{g}(N)$ is the ground-state energy for $N$ particles in the system. Since $\left|\Psi_{g}\left(N<N_{\Lambda}\right)\right\rangle$ is a wave function with nonzero norm, this relation means $E_{g}(N)=0$. As a consequence, for $N \leqslant N_{\Lambda}$ -1 , we have for $\mu^{+}=E_{g}(N+1)-E_{g}(N)$ and $\mu^{-}=E_{g}(N)$ $-E_{g}(N-1)$, the expression

$$
\mu^{+}-\mu^{-}=0 .
$$


Therefore, the state we analyze is conducting (see also Ref. 56).

Furthermore, the $\hat{S}_{i}^{z} \hat{S}_{j}^{z}$ operator will have nonzero matrix elements only along the diagonal in $R_{N}$ variables, and in conditions mentioned for $p_{i}$ after Eq. (23) we further have $\left\langle\left\langle\hat{S}_{i}^{z} \hat{S}_{j}^{z}\right\rangle\right\rangle=0$.

As can be seen, Eqs. (18) and (19) describe a localizationdelocalization transition driven by the electron concentration $\rho_{n}$, which emerges at $\rho_{n}^{c}=1 / 4$, the delocalized phase being present in the region $\rho_{n}<\rho_{n}^{c}$. The occurrence of this transition is intimately connected to the multielectronic nature of the description which is made in the presence of the interparticle interaction and absence of trapping centers. Indeed, the problem considered at the level of independent electron approximation (e.g., absence of interelectronic interaction) in the presence of trapping centers leads to a one-particle problem in the presence of an attractive potential, which ends up usually at small energies with localization. Here all these are avoided.

Concerning the possibility of the emergence of Griffiths phases in influencing the described transition, we mention that the Griffiths singularities arise due to the presence of statistically rare clusters that are anomalously strongly coupled, and hence they are unique features of the disordered system (see, for example, Ref. 57). The effect becomes weaker with increasing dimension, increasing interaction, and increasing number of the components $\bar{N}$ of the dynamical variables. In our case $\bar{N}=3$ (for example, for spin), the dimension of the (quantum mechanical) description is $D$ $=2$, and the results are not valid at zero interelectronic in- teraction. All these conditions make unlikely the major influence of Griffiths phases, especially when the $p_{i}$ parameters are all maintained perfectly random at all sites as mentioned below Eq. (23), prohibiting in this way the local formation of anomalously strongly coupled clusters.

\section{SUMMARY AND CONCLUSIONS}

We deduced exact multielectronic concentration dependent ground states for disordered and interacting twodimensional quantum-mechanical systems at and below quarter filling. The ground states describe a localizationdelocalization transition driven by concentration and provide paramagnetic behavior. The ground-state nature is lost in the absence of the interaction, e.g., independent electron approximation. The deduced results are nonperturbative and cannot be perturbatively reached from the noninteracting, although disordered case. The studied system is of two-band type, and the disorder is present independently in both $\hat{H}_{\text {int }}$ and $\hat{H}_{0}$ parts of the Hamiltonian, the trapping centers being excluded. The presented procedure extends the exact solution possibilities known in 2D Penrose lattice case to nonquasicrystalline disordered systems as well, even in the presence of the interelectronic interactions.

\section{ACKNOWLEDGMENTS}

The research was supported by the Hungarian Fund for Scientific Research through Contract Nos. OTKA-T-037212 and FKFP-0471. The author thanks Florian Gebhard for valuable discussions relating to metal-insulator transitions.
${ }^{1}$ P.A. Lee and T.V. Ramakrishnan, Rev. Mod. Phys. 57, 287 (1985).

${ }^{2}$ J.D. Maynard, Rev. Mod. Phys. 73, 401 (2001).

${ }^{3}$ S.V. Kravchenko, G.V. Kravchenko, J.E. Furneaux, V.M. Pudalov, and M. D'Iorio, Phys. Rev. B 50, 8039 (1994).

${ }^{4}$ E. Abrahams, P.W. Anderson, D.C. Licciardello, and T.V. Ramakrishnan, Phys. Rev. Lett. 42, 673 (1979).

${ }^{5}$ E. Abraham, S.V. Kravchenko, and M.P. Sarachik, Rev. Mod. Phys. 73, 251 (2001).

${ }^{6}$ S. Bogdanovich and D. Popovic, Physica E (Amsterdam) 12, 604 (2002).

${ }^{7}$ S. Gupta, S. Sil, and B. Bhattacharyya, cond-mat/0212515 (unpublished).

${ }^{8}$ V. Dobrosavljevic, D. Tanakovic, and A.A. Pastor, Phys. Rev. Lett. 90, 016402 (2003).

${ }^{9}$ A. Ghosal, M. Randeria, and N. Trivedi, Phys. Rev. B 65, 014501 (2001).

${ }^{10}$ D. Belitz and T.R. Kirkpatrick, Rev. Mod. Phys. 66, 261 (1994).

${ }^{11}$ B.J. Powell, J.F. Annett, and B.L. Györffy, cond-mat/0301364 (unpublished).

${ }^{12}$ Z. Gulácsi and M. Gulácsi, Phys. Rev. Lett. 73, 3239 (1994).

${ }^{13}$ Z. Gulácsi and M. Gulácsi, Phys. Rev. B 40, 708 (1989).

${ }^{14}$ D.K. Morr and N.A. Stavropoulos, Phys. Rev. B 67, 020502(R) (2003)
${ }^{15}$ M. Kohmoto, L.P. Kadanoff, and C. Tang, Phys. Rev. Lett. 50, 1870 (1983).

${ }^{16}$ D.R. Grempel, S. Fishman, and R.E. Prange, Phys. Rev. Lett. 49, 833 (1982).

${ }^{17}$ U. Grimm and M. Schreiber, cond-mat/0212140 (unpublished).

${ }^{18}$ U. Grimm, R.A. Römer, and G. Schliecker, Ann. Phys. (Leipzig) 7, 389 (1998).

${ }^{19}$ D. Levine and P.J. Steinhardt, Phys. Rev. Lett. 53, 2477 (1984).

${ }^{20}$ S. Ostlund, R. Pandit, H.J. Schellnhuber, and E. Siggia, Phys. Rev. Lett. 50, 1873 (1983).

${ }^{21}$ M. Kohmoto, B. Sutherland, and C. Tang, Phys. Rev. B 35, 1020 (1987).

${ }^{22}$ R. Penrose, Bull. Inst. Math. Appl. 10, 266 (1974).

${ }^{23}$ M. Gardner, Sci. Am. 236, 110 (1977).

${ }^{24}$ M. Kohmoto and B. Sutherland, Phys. Rev. B 34, 3849 (1986).

${ }^{25}$ B. Sutherland, Phys. Rev. B 34, 3904 (1986).

${ }^{26}$ M. Arai, T. Tokihiro, T. Fujiwara, and M. Kohmoto, Phys. Rev. B 38, 1621 (1988).

${ }^{27}$ P. Repetowitcz, U. Grimm, and M. Schreiber, Phys. Rev. B 58, 13482 (1998).

${ }^{28}$ R.G. Hennig, K.F. Kelton, A.E. Carlsson, and C.L. Henley, Phys. Rev. B 67, 134202 (2003).

${ }^{29}$ E.Y. Vedmedenko, H.P. Oepen, and J. Kirschner, Phys. Rev. Lett. 90, 137203 (2003). 
${ }^{30}$ M. Kollar, R. Strack, and D. Vollhardt, Phys. Rev. B 53, 9225 (1996).

${ }^{31}$ M. Gulácsi, Philos. Mag. B 76, 731 (1997).

${ }^{32}$ O.O. Bernal, D.E. MacLaughlin, H.G. Lukefahr, and B. Andraka, Phys. Rev. Lett. 75, 2023 (1995).

${ }^{33}$ E. Miranda, V. Dobrosavljevic, and G. Kotliar, Phys. Rev. Lett. 78, 290 (1997).

${ }^{34}$ E. Miranda and V. Dobrosavljevic, Phys. Rev. Lett. 86, 264 (2001)

${ }^{35}$ J.C. Cooley, M.C. Aronson, Z. Fisk, and P.C. Canfield, Phys. Rev. Lett. 74, 1629 (1995).

${ }^{36}$ Z. Gulácsi and I. Orlik, J. Phys. A 34, L359 (2001).

${ }^{37}$ I. Orlik and Z. Gulácsi, Philos. Mag. B 81, 1587 (2001).

${ }^{38}$ L.G. Sarasua and M.A. Continentino, Phys. Rev. B 65, 233107 (2002).

${ }^{39}$ P. Gurin and Z. Gulácsi, Phys. Rev. B 64, 045118 (2001); 65, 129901(E) (2002).

${ }^{40}$ Z. Gulácsi, Phys. Rev. B 66, 165109 (2002).

${ }^{41}$ Z. Gulácsi, Eur. Phys. J. B 30, 295 (2002).

${ }^{42}$ Z. Gulácsi and D. Vollhardt, Phys. Rev. Lett. 91, 186401 (2003).

${ }^{43}$ W. Lu, Z. Shu, J. Dong, and E.G. Wang, Phys. Rev. B 66, 245105 (2002).
${ }^{44}$ G.L. Caer, J. Phys. A 24, 4655 (1991).

${ }^{45}$ T. Hotta, cond-mat/0212575 (unpublished).

${ }^{46}$ Y. Asada, K. Slevin, and T. Ohtsuki, Phys. Rev. Lett. 89, 256601 (2002).

${ }^{47}$ W. Koller et al., cond-mat/0301350 (unpublished).

${ }^{48}$ R. Schorr and H. Rieger, cond-mat/0212526 (unpublished).

${ }^{49}$ J. Staring, B. Mehlig, Y.V. Fyodorov, and J.M. Luck, cond-mat/0301127 (unpublished).

${ }^{50}$ K. Takeda and I. Ichinose, Nucl. Phys. B 663, 520 (2003).

${ }^{51}$ J. Lemaitre et al., Philos. Mag. B 67, 347 (1993).

${ }^{52}$ D. Shechtman, I. Blech, D. Gratias, and J.W. Cahn, Phys. Rev. Lett. 53, 1951 (1984).

${ }^{53}$ K. Semba and T. Ninomiya (unpublished) (see references from Ref. 26).

${ }^{54}$ T. Fujiwara, M. Arai, T. Tokihiro, and M. Kohmoto, Phys. Rev. B 37, 2797 (1988).

${ }^{55}$ F. Gebhard, The Mott Metal-Insulator Transition, Springer Tracts in Modern Physics Vol. 137 (Springer-Verlag, Berlin, 1997), p. 6.

${ }^{56}$ E.H. Lieb and F.Y. Wu, Phys. Rev. Lett. 20, 1445 (1968).

${ }^{57}$ S. Sachdev, Quantum Phase Transitions (Cambridge University Press, Cambridge, 1999), p. 302. 